\title{
Osteopontin versus alpha-fetoprotein as a diagnostic marker for hepatocellular carcinoma: a meta-analysis
}

This article was published in the following Dove Press journal: OncoTargets and Therapy

\author{
Tingting Sun ${ }^{1, *}$ \\ Yurong Tangl,* \\ Diwen Sun ${ }^{2}$ \\ Qingao $\mathrm{Bu}^{2}$ \\ Peng $\mathrm{Li}^{2}$
}

'Department of Clinical Laboratory, Shengli Oilfield Central Hospital, Dongying 257000, Shandong, China; ${ }^{2}$ Department of Breast and Thyroid Surgery, Shengli Oilfield Central Hospital, Dongying 257000, Shandong, China

*These authors contributed equally to this work
Correspondence: Peng Li

Department of Breast and Thyroid Surgery, Shengli Oilfield Central Hospital, No 3I, Jinan Rd, Dongying 257000,

Shandong, China

Tel +86546877 090 I

Fax +8654687I 7968

Email lipeng.good1987@I63.com
Background: Several studies have reported that osteopontin (OPN) is a promising marker for the diagnosis of hepatocellular carcinoma (HCC); however, some studies emerged with conflicting results. Therefore, we provide a systematic review to evaluate the diagnostic performance of OPN for HCC.

Methods: Studies that investigated the diagnostic value of OPN and alpha-fetoprotein (AFP) in HCC were collected from PubMed and Embase. Sensitivity, specificity, and other parameters about the diagnostic accuracy of serum OPN and AFP in HCC were pooled using STATA 12.0 software. The summary receiver operating characteristic curve (sROC) and other parameters were used to summarize the overall test performance.

Results: Twelve studies were included in our meta-analysis. Pooled sensitivity, specificity, and diagnostic odds ratio were 0.813 (95\% CI: 0.671-0.902), 0.874 (95\% CI: $0.778-0.932$ ), and 30.047 (95\% CI: 8.845-102.067) for OPN; 0.639 (95\% CI: $0.538-0.729), 0.959$ (95\% CI: 0.909-0.982), and 41.518 (95\% CI: 13.688-125.929) for AFP; and 0.856 (95\% CI: 0.760-0.918), 0.738 (95\% CI: 0.630-0.823), and 16.718 (95\% CI: 7.950-35.156) for OPN+AFP, respectively. The area under the sROC for OPN, AFP, and OPN+AFP was $0.91,0.88$, and 0.85 , respectively. For diagnosis of early HCC, pooled sensitivity of serum OPN, AFP, and OPN+AFP was 0.493 (95\% CI: $0.422-0.563), 0.517$ (95\% CI: 0.446-0.587), and 0.732 (95\% CI: $0.666-0.791)$, respectively.

Conclusions: OPN is a comparable marker to AFP for the diagnosis of HCC, and the sensitivity of OPN was higher than that of AFP. A combination of AFP and OPN can elevate the sensitivity of diagnosis for early HCC.

Keywords: OPN, AFP, HCC, diagnosis, meta-analysis

\section{Introduction}

Hepatocellular carcinoma (HCC) is one of the most aggressive cancers worldwide as well as the third cause of cancer-related mortality. ${ }^{1,2}$ Because most patients are not diagnosed until the disease progresses to advanced liver cancer and the prognosis of HCC is generally poor, the 5-year survival rate is $<10 \%-15 \%{ }^{3,4}$ Early detection of liver cancer can give an opportunity for surgical resection and transplantation, which can benefit a number of patients. Therefore, it is particularly important to diagnose HCC early and accurately.

Generally, the diagnostic methods for HCC include blood chemistry tests like alpha-fetoprotein (AFP) and imaging such as computed tomography (CT), magnetic resonance imaging (MRI), and ultrasound. ${ }^{5}$ However, although ultrasound is an indirect diagnosis method, which is convenient and noninvasive, its accuracy depends largely 
on the skill of the operator, and the ability to distinguish HCC from non-neoplastic nodules is limited. ${ }^{6} \mathrm{CT}$ and MRI can produce higher resolution images than ultrasonography, but they are more expensive and $\mathrm{CT}$ is related to radiation exposure. ${ }^{7}$ Furthermore, for small and well-differentiated lesions, the sensitivity of CT and MRI was lower. Presently, AFP is widely used in the diagnosis of HCC. However, the diagnostic performance is not ideal because its sensitivity only ranges from $40 \%$ to $65 \%{ }^{8,9}$ Therefore, it is particularly important to identify a new biomarker with superior diagnostic accuracy.

Osteopontin (OPN) is a multifunctional phosphorylated glycoprotein, which could be expressed in a variety of cell types including $\mathrm{T}$ lymphocytes, macrophages, dendritic cells, and osteoclasts. ${ }^{10-12}$ Recent studies have reported that OPN overexpression has been detected in lung cancer, breast cancer, and HCC. ${ }^{13-15}$ Two major studies have identified that OPN as a promising biomarker for the diagnosis of $\mathrm{HCC}$ had greater performance than AFP in distinguishing HCC cases from liver cirrhosis patients. ${ }^{15,16}$ Furthermore, Shang et $\mathrm{al}^{15}$ found that the level of serum OPN was already increased a year prior to HCC diagnosis, indicating that OPN is a promising diagnostic marker for HCC. Although many studies have reported that serum OPN is a biomarker for $\mathrm{HCC}$, the diagnostic value of serum OPN is still debatable. ${ }^{17,18}$ Therefore, we systematically reviewed studies to evaluate the diagnostic accuracy of serum OPN by directly comparing with AFP for HCC diagnosis.

\section{Methods}

\section{Identification and selection of studies}

Studies were identified by searching PubMed and Embase covering all papers published up to June 1, 2018. The search strategy used the following terms: (OPN or SPP1 [MESH] or OPN or SPP1 [TEXT WORD]) AND (carcinoma, hepatocellular [MESH] or HCC [TEXT WORD]). Studies in English were eligible for inclusion. Additionally, the references were also checked for triage of additional suitable articles, including all the identified studies and reviews. Once studies were found with overlapping data published by the same research center, only the publication with most complete data was included. All the studies were reviewed by two reviewers independently based on titles and abstracts, and then the full texts of potentially eligible studies were retrieved for further assessment. When the final result was inconsistent, our group discussed it according to the inclusion and exclusion criteria.

\section{Inclusion criteria of studies}

Eligible studies in this meta-analysis should meet the following criteria: 1) the included studies must be published in English language; 2) the diagnosis of HCC was based on histological examination or the appropriate imaging characteristics as defined by accepted guidelines; 3) studies evaluated the diagnosis accuracy of serum OPN and AFP for HCC in the same patients; 4) serum samples were used to test OPN and AFP; 5) the sensitivity and specificity of OPN and AFP were provided or could be calculated; and 6) editorial letters, conference abstracts, reviews, case reports, and comments were excluded.

\section{Data extraction}

The studies' information for this meta-analysis was retrieved by the reporting checklists of Preferred Reporting Items for Systematic Reviews and Meta-analysis (PRISMA) guidelines. Data extracted from the studies included the following items: 1) publication information (first author's last name, year of publication); 2) patients' characteristic information (study population and regions, age, gender, number of patient); and 3) OPN and AFP information (methods, cutoff values, and raw data results (number of true-positive, falsenegative, false-positive and true-negative)).

\section{Assessment of methodological quality}

The Quality Assessment of Diagnostic Accuracy Studies-2 (QUADAS-2) was used to assess the quality of included studies. ${ }^{19}$ Two authors independently assessed the quality of included studies according to the QUADAS-2.

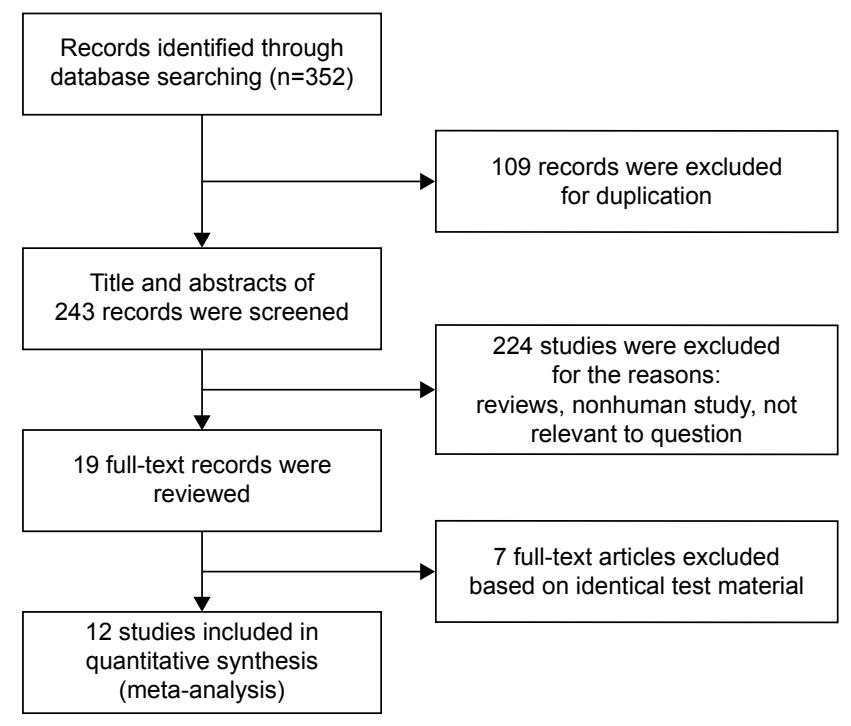

Figure I Flowchart of the study selection strategy. 


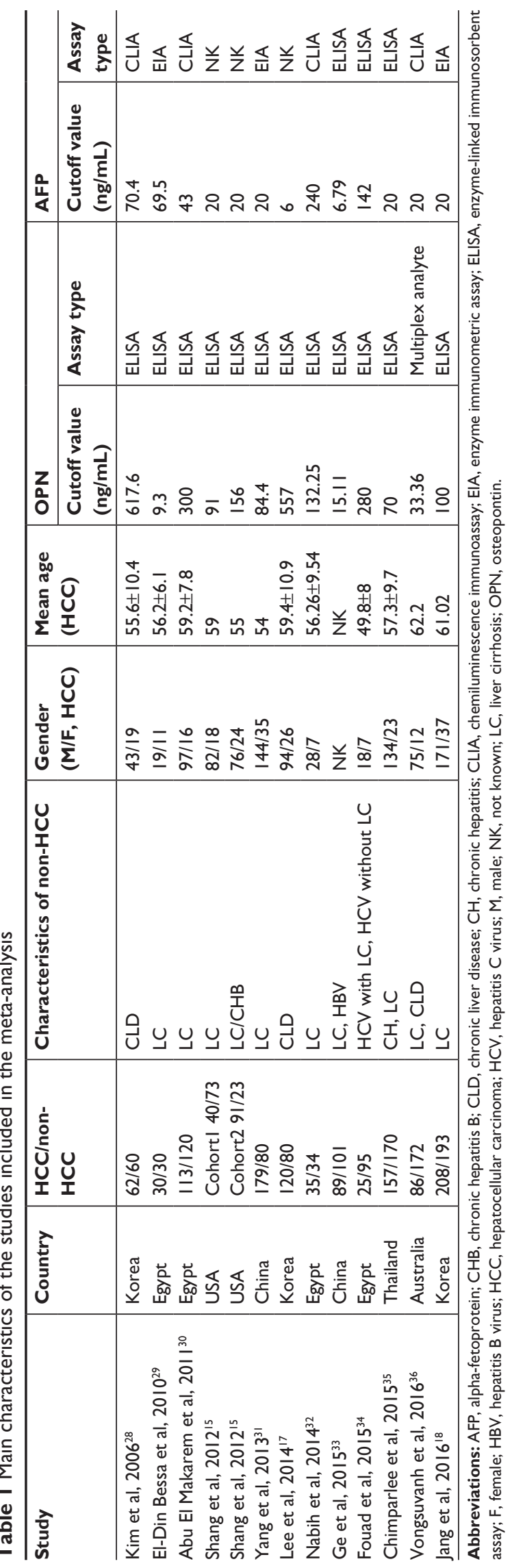

The QUADAS-2 tool contains four domains: patient selection, index test, reference standard, and flow and timing. Each of the signaling questions used for judging the risk of bias was labeled as "yes", "no", or "unclear". Each of the domains that assessed the risk of bias and concerns about applicability were rated as high risk, low risk, and unclear risk. If no consensus was reached, a third author was an arbitrator.

\section{Statistical analysis}

Statistical analyses were performed by Meta-Disc (version 1.4) and STATA version 12.0 (StataCorp LP, College Station, TX, USA). The sensitivity and specificity in diagnosis of HCC were calculated using the bivariate model. ${ }^{20}$ Heterogeneity of pooled results was assessed using the $\mathrm{Q}$ statistic and I-squared index. We reported the summary results in terms of sensitivity, specificity, negative and positive likelihood ratios, and diagnostic odds ratios (DORs). Forest plots and summary receiver operating characteristic curves (sROCs) were used

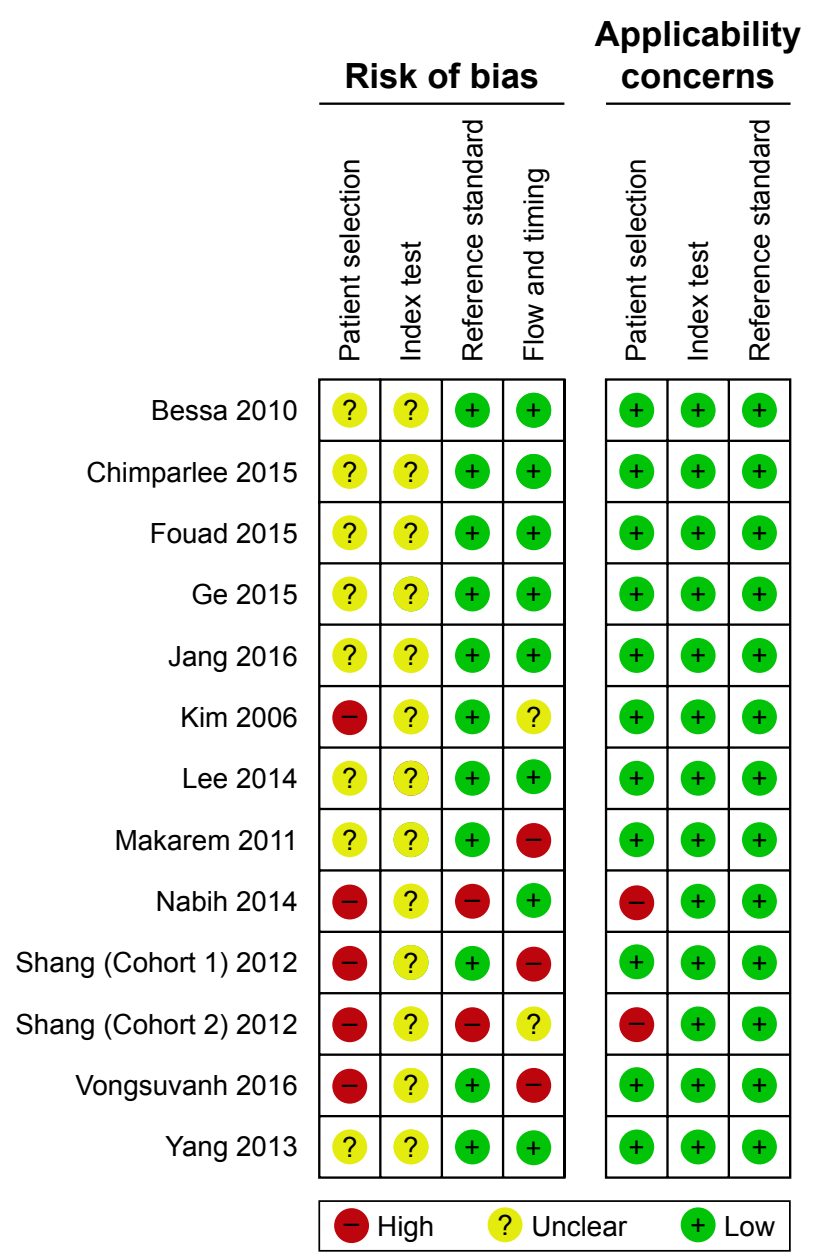

Figure 2 Summary assessment of methodological quality of included studies by Quality Assessment of Diagnostic Accuracy Studies-2. 
to visualize the summary results. Univariate regression for sensitivity and specificity was performed to explore the possible covariates of heterogeneity. Deeks' analysis was applied to evaluate the publication bias. ${ }^{21}$

\section{Results}

\section{Study selection}

The initial search identified 352 relevant papers, of which 109 were duplicates. After title and abstract review, 19 studies were selected for detailed evaluation. Seven studies were excluded after full assessment due to lacking sufficient data to calculate the sensitivity and specificity, or they were not diagnostic studies or overlapped studies. ${ }^{16,22-27}$ Finally, 12 studies $^{15,17,18,28-36}$ were included in our meta-analysis, including 1,235 patients with HCC and 1,191 controls. The flowchart of study selection is shown in Figure 1. All patients received a single serum OPN and AFP test. Five of the 12 studies included $705 \mathrm{HCC}$ patients and 600 controls who
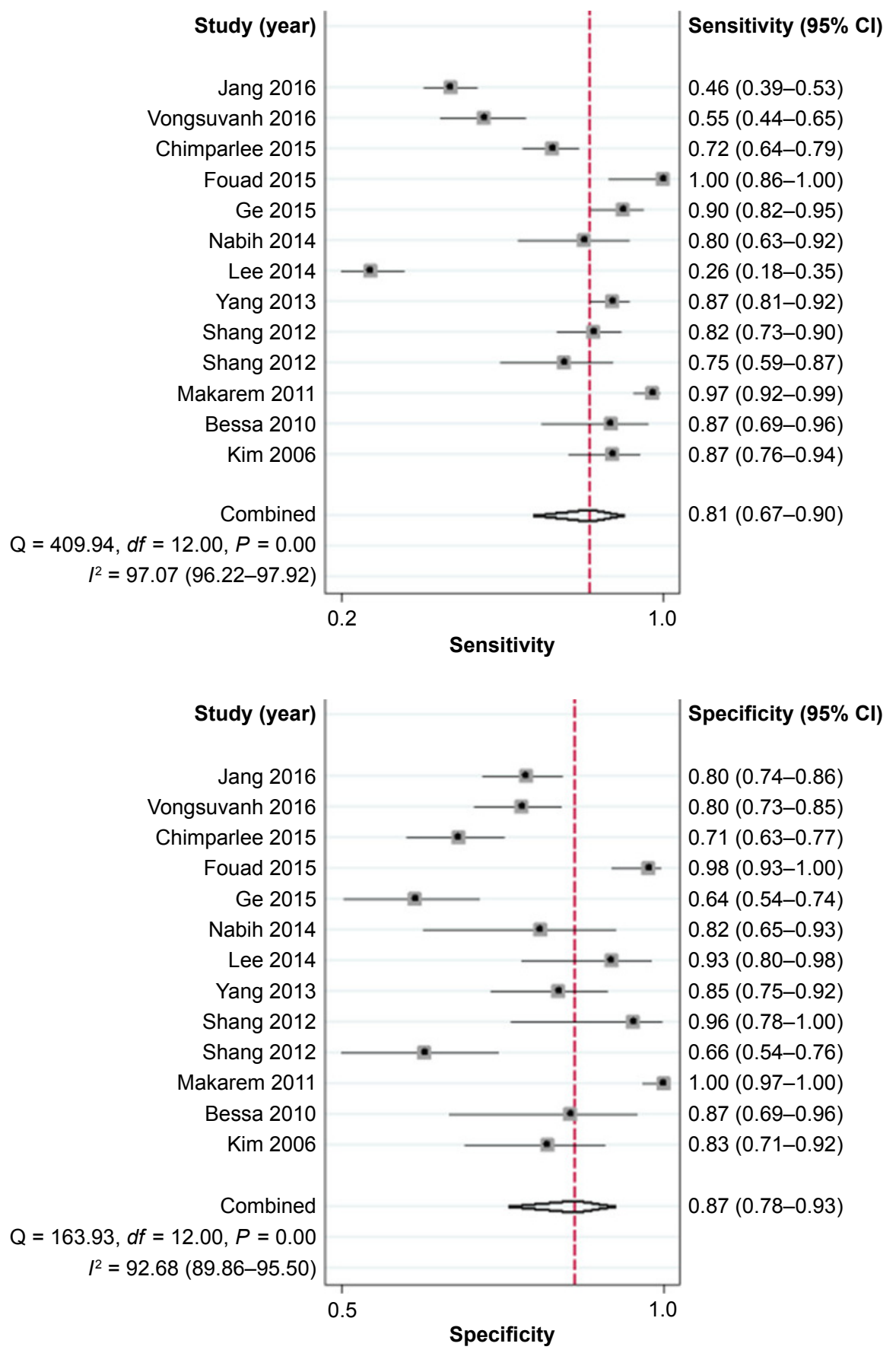

Figure 3 Forest plots of the sensitivity and specificity of osteopontin in the diagnosis of hepatocellular carcinoma. 
underwent combined serum OPN and AFP tests. ${ }^{15,17,33,35,36}$ The characteristics of included studies are shown in Table 1.

\section{Quality of the studies}

The quality evaluation of the eligible studies is shown in Figure 2. All articles were retrospective studies, and none have reported that the patients enrolled were a consecutive or random sample. Thus, the patient selection domain in these studies was labeled as "unclear". We considered the risk of bias in four studies ${ }^{15,28,32,36}$ and applicability concerns in two studies ${ }^{15,32}$ were labeled as "high" due to inadequate exclusions. Concerning the index test, the risks of bias were all labeled as "unclear" because the studies did not report whether the threshold was prespecified. Regarding the reference standard, we considered it to be of high risk of bias if based on imaging (ultrasonography and CT) and/or
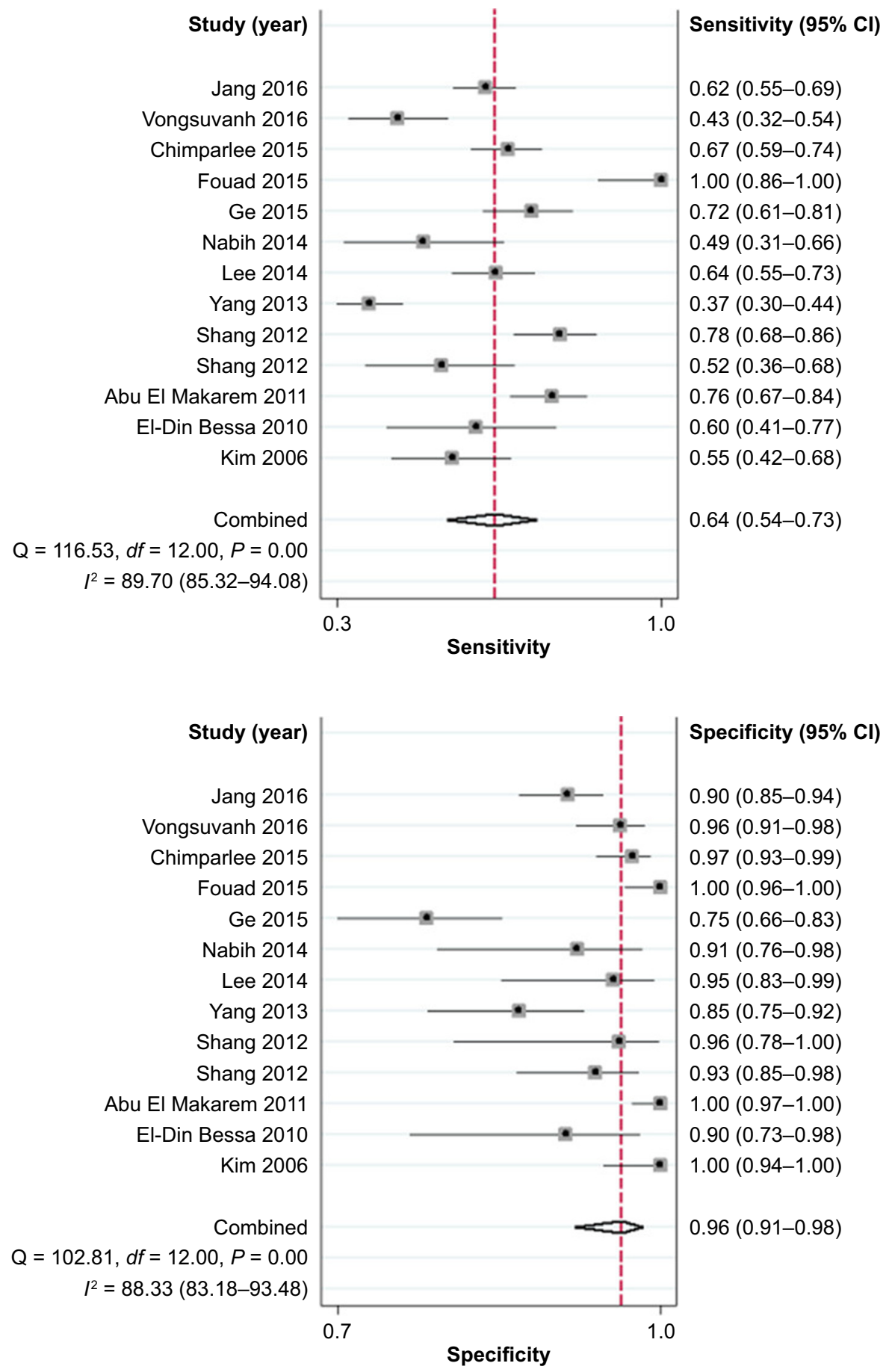

Figure 4 Forest plots of the sensitivity and specificity of alpha-fetoprotein in the diagnosis of hepatocellular carcinoma. 
biochemistry (AFP) to independently identify HCC. ${ }^{15,17,30,32}$ For the flow and timing domain, two studies were labeled as "unclear" as there were no reports showing whether all patients received the same reference standard. ${ }^{15,28}$ Because three studies reported patients diagnosed with HCC by two different reference standards (histologic examination and imaging patterns), we considered this to be a high risk of bias. ${ }^{15,18,29}$

\section{Diagnostic accuracy of OPN and AFP for HCC}

The sensitivity and specificity of OPN and AFP for diagnosing HCC are shown in Figures 3 and 4. The pooled sensitivity and specificity of serum OPN were $81.3 \%$ (95\% CI: $67.1 \%-90.2 \%$ ) and $87.4 \%$ (95\% CI: 77.8\%-93.2\%), respectively. For AFP, pooled sensitivity and specificity were 63.9\% (95\% CI: 53.8\%-72.9\%) and 95.9\% (95\% CI: 90.9\%$98.2 \%)$, respectively. The DOR incorporated sensitivity and specificity into a single indicator and was not influenced by the morbidity of disease. ${ }^{37}$ The pooled DOR in our study for

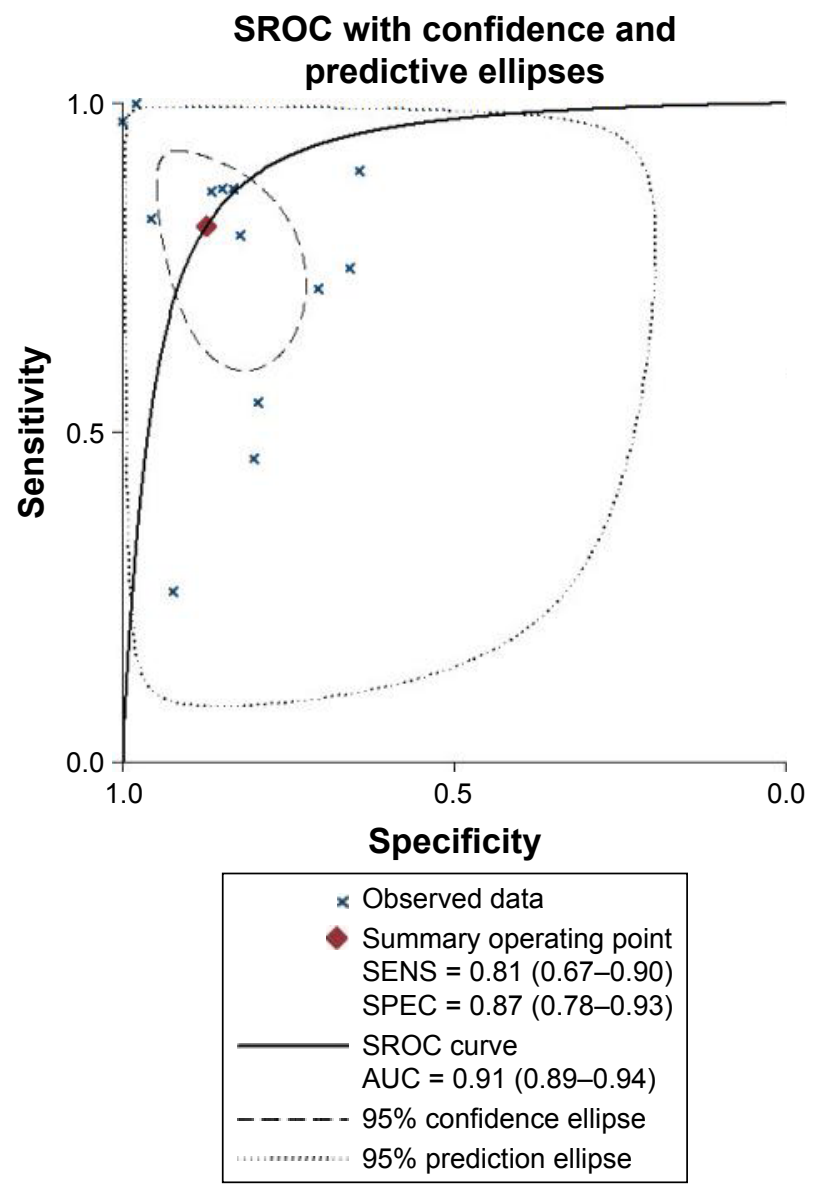

Figure 5 SROC curve of osteopontin for diagnosing hepatocellular carcinoma. AUC: 0.91.

Abbreviations: AUC, area under the curve; SENS, sensitivity; SPEC, specificity; SROC, summary receiver operating characteristic.
OPN and AFP was 30.047 (95\% CI: 8.845-102.067) and 41.518 (95\% CI: 13.688-125.929), respectively. The sROC curves of OPN and AFP are shown in Figures 5 and 6. The area under the curve (AUC) was 0.91 (95\% CI: 0.89-0.94) for OPN and 0.88 (95\% CI: 0.85-0.90) for AFP. The sROC curve of OPN was close to the AFP curve, which indicates that OPN had comparable diagnosis accuracy to AFP for HCC $(P>0.05)$. All results are shown in Table 2.

\section{Serum OPN combined with AFP for the diagnosis of HCC}

Five studies ${ }^{15,17,33,35,36}$ have reported the diagnostic accuracy of serum OPN combined with AFP for HCC. Results are shown in Figures 7 and 8, and Table 2. The pooled sensitivity and specificity were $85.6 \%(95 \% \mathrm{CI}: 76.0 \%-91.8 \%)$ and 73.8\% (95\% CI: 63.0\%-82.3\%), respectively. Compared with OPN and AFP, the sensitivity value of OPN+AFP was superior to that of each marker alone. The DOR for serum OPN combined with AFP was $16.718(95 \%$ CI: 7.950-35.156). The DOR was significantly lower

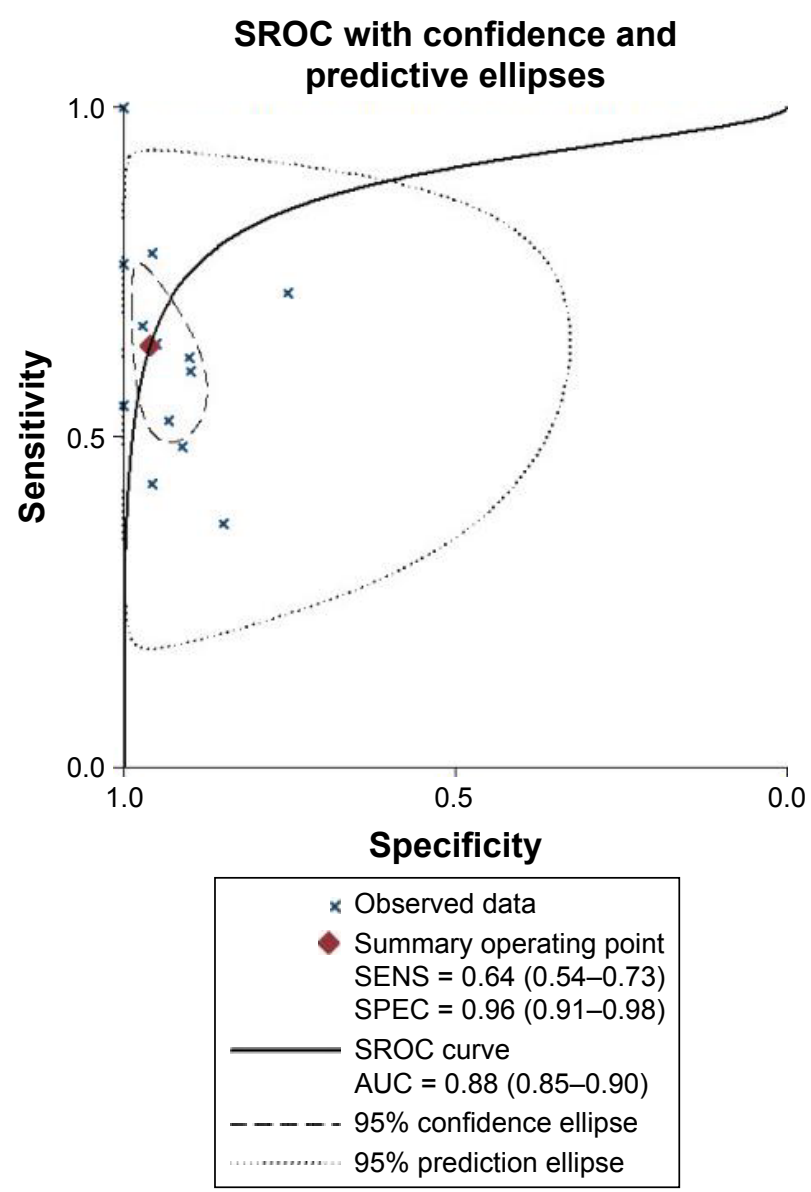

Figure 6 Summary receiver operating characteristic (SROC) curve of alphafetoprotein for diagnosing hepatocellular carcinoma. Area under the curve (AUC): 0.88 . Abbreviations: AUC, area under the curve; SENS, sensitivity; SPEC, specificity; SROC, summary receiver operating characteristic. 
Table 2 Summary of the diagnostic accuracy of OPN, AFP, and OPN+AFP

\begin{tabular}{|c|c|c|c|c|c|c|}
\hline Marker & Pooled sensitivity & Pooled specificity & PLR & NLR & DOR & AUC \\
\hline OPN & $0.8 \mid 3(0.67 \mid-0.902)$ & $0.874(0.778-0.932)$ & $6.436(3.299-12.553)$ & $0.2 \mid 4(0.1|I|-0.4 \mid 3)$ & 30.047 (8.845-102.067) & $0.91(0.89-0.94)$ \\
\hline AFP & $0.639(0.538-0.729)$ & $0.959(0.909-0.982)$ & $15.6 \mid 8$ (6.384-38.209) & $0.376(0.283-0.501)$ & 41.518 (13.688-125.929) & $0.88(0.85-0.90)$ \\
\hline OPN+AFP & $0.856(0.760-0.918)$ & $0.738(0.630-0.823)$ & $3.266(2.257-4.727)$ & $0.195(0.115-0.333)$ & $16.718(7.950-35.156)$ & $0.85(0.82-0.88)$ \\
\hline
\end{tabular}

Abbreviations: AFP, alpha-fetoprotein; AUC, area under curve; DOR, diagnostic odds ratio; NLR, negative likelihood ratio; OPN, osteopontin; PLR, positive likelihood ratio.

compared to OPN or AFP alone $(P<0.05)$. The AUC of Summary of diagnostic accuracy of serum the sROC for OPN combined with AFP was 0.85 (95\% CI: OPN and AFP for early HCC

0.82-0.88) and has no difference compared with OPN or Four studies ${ }^{31,33,35,36}$ reported the diagnostic accuracy of serum AFP alone $(P>0.05)$.

OPN and AFP for early HCC (Barcelona Clinic Liver Cancer
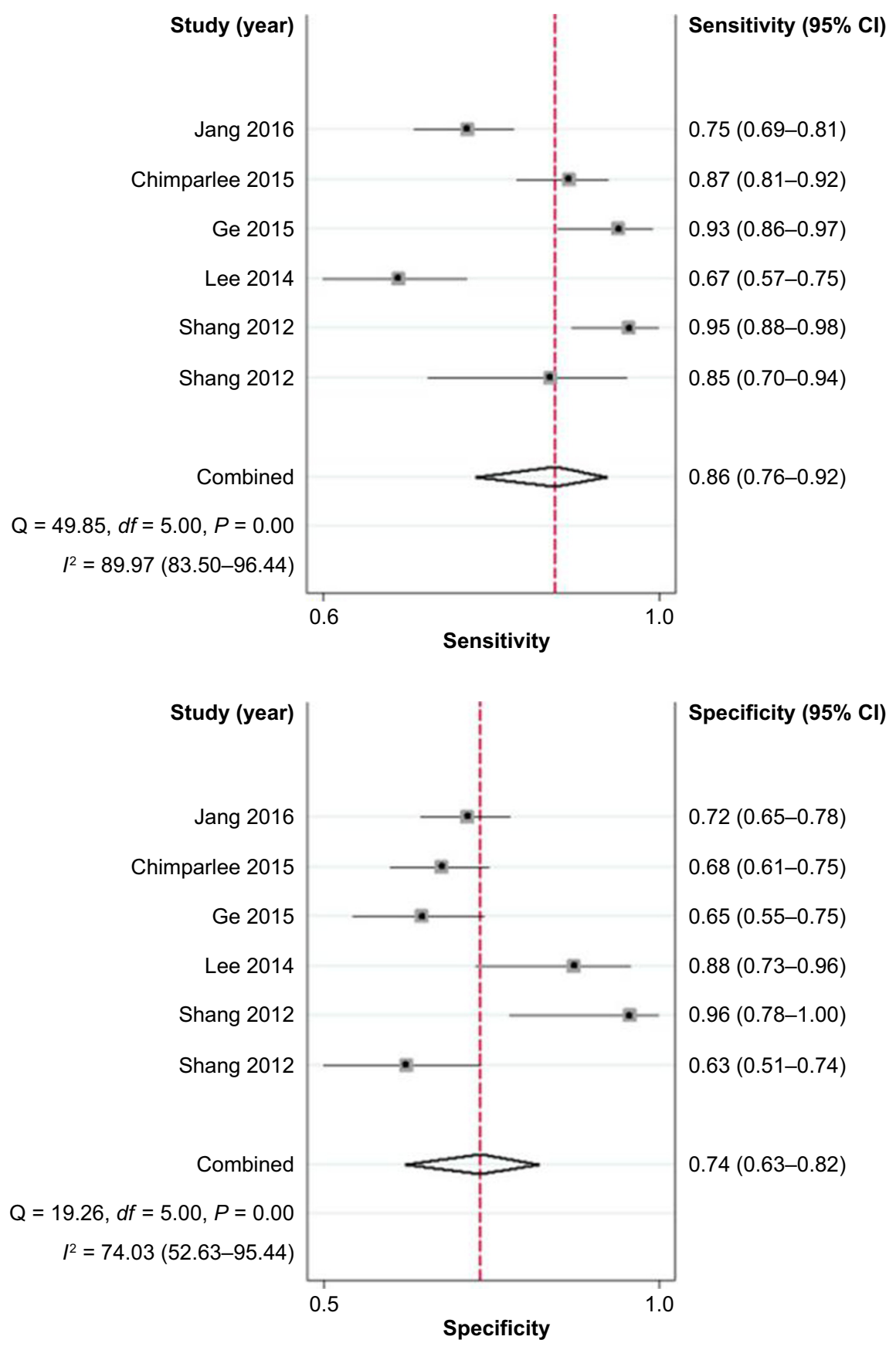

Figure 7 Forest plots of the sensitivity and specificity of osteopontin+alpha-fetoprotein in the diagnosis of hepatocellular carcinoma. 


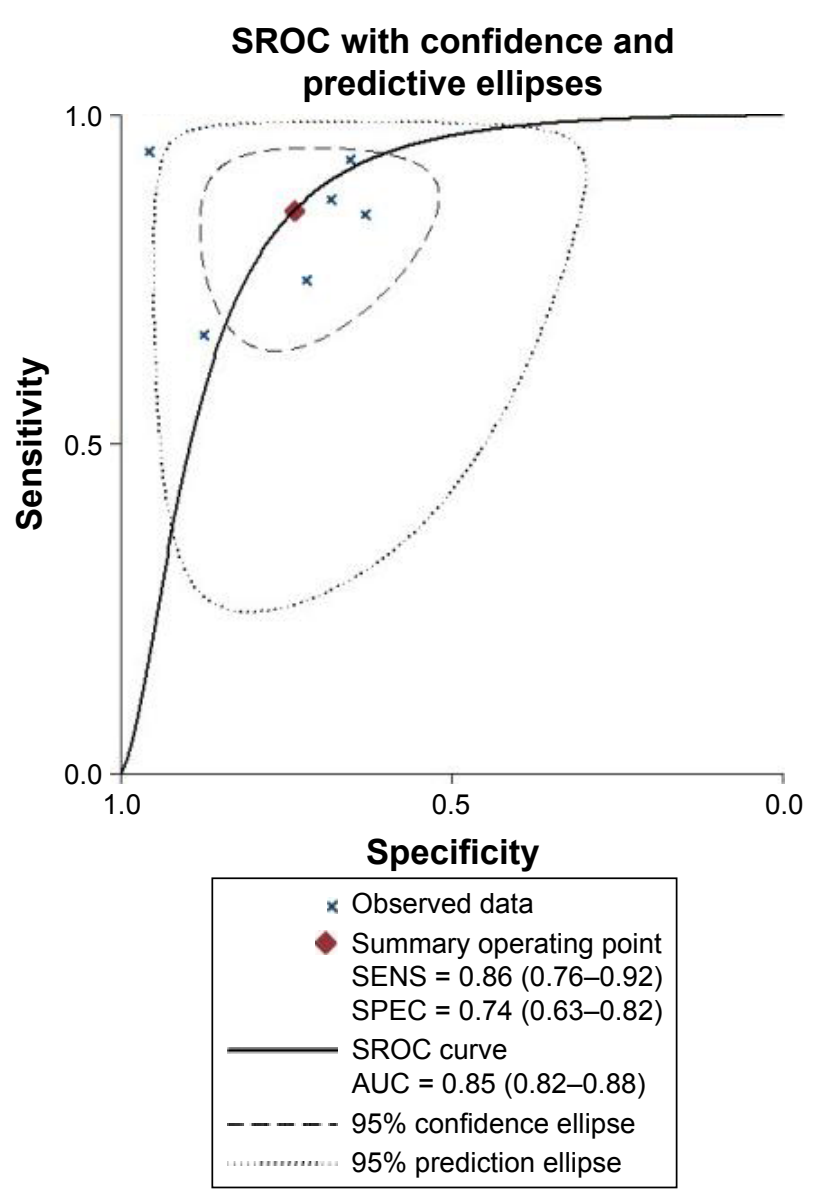

Figure 8 SROC curve of osteopontin+alpha-fetoprotein for diagnosing hepatocellular carcinoma. AUC: 0.85 .

Abbreviations: AUC, area under the curve; SENS, sensitivity; SPEC, specificity; SROC, summary receiver operating characteristic.

Classification 0-A). As the "metandi" command in STATA requires data from more than four studies, we used Meta-Disc (version 1.4). The pooled sensitivity and specificity of serum OPN for diagnosis of early HCC were 0.493 (95\% CI: $0.422-$ 0.563 ) and 0.717 (95\% CI: 0.677-0.755), respectively. For AFP, pooled sensitivity and specificity were 0.517 (95\% CI: 0.446-0.587) and 0.899 (95\% CI: 0.871-0.924), respectively, while the pooled sensitivity and specificity for AFP combined with OPN were 0.732 (95\% CI: 0.666-0.791) and 0.683 (95\% CI: 0.642-0.723), respectively. The DOR and 95\% CI for OPN and AFP were 3.667 (95\% CI: 1.136-11.842) and 9.710 (95\% CI: 5.490-17.175), respectively. Combination of AFP and OPN can elevate the sensitivity of diagnosis for early HCC. The diagnostic value of serum OPN for early HCC also needs further investigation in future studies.

\section{Investigation for heterogeneity and publication bias}

The heterogeneity of sensitivity and specificity was significant among included studies. We attempted to explore the
Table 3 Meta-regression analysis of the effects of OPN and AFP on diagnostic accuracy

\begin{tabular}{l|l|l|l|l}
\hline Variable & \multicolumn{2}{l}{ OPN } & \multicolumn{2}{l}{ AFP } \\
\cline { 2 - 5 } & Coefficient & $P$-value & Coefficient & $P$-value \\
\hline Race & 1.848 & 0.1386 & 0.382 & 0.7203 \\
Cutoff & 1.408 & 0.3514 & 1.493 & 0.2438 \\
Method & -1.786 & 0.4562 & -1.430 & 0.3766 \\
\hline
\end{tabular}

Abbreviations: AFP, alpha-fetoprotein; OPN, osteopontin.

reasons for heterogeneity by analyzing the study characteristics using meta-regression. The result is shown in Table 3. However, possibly because of the limited number of studies included, the differences of races, cutoff value, and assay type did not have a statistically significant effect on the diagnostic accuracy. Moreover, we used Deeks' funnel plot to analyze potential publication bias and it showed some asymmetry (Figure 9). However, the $P$-values for Deeks' test were 0.411 for AFP, 0.565 for OPN, and 0.149 for OPN combined with AFP for diagnosing HCC, and this suggested that there was no publication bias in our meta-analysis.

\section{Discussion}

After carefully retrieving literature, we selected 12 studies to evaluate the diagnostic accuracy of serum OPN and AFP for HCC in this meta-analysis. Finally, four studies clearly stated serum OPN performed better than AFP, ${ }^{17,18,35,36}$ while the remaining studies had the opposite conclusion or no clear results. Pooled sensitivity and specificity of OPN for HCC was 0.813 and 0.874 , respectively. The DOR was 30.047 and the AUC of the sROC was 0.91. All these results showed the moderate diagnostic accuracy of OPN in HCC. To our knowledge, two studies have performed a systematic review about the serum OPN as a biomarker for HCC diagnosis. . $^{38,39}$ However, Cheng et al ${ }^{38}$ performed a meta-analysis on merely four studies and the evidence indicates that plasma OPN had a comparable accuracy with AFP for HCC diagnosis. After analyzing seven studies, Wan et al ${ }^{39}$ suggested that OPN is a comparable marker to AFP for the diagnosis of HCC, but the combination value of OPN and AFP still needed further research. Compared with the previous meta-analysis, our review included more patients and studies; furthermore, we included some conflicting studies and performed the combination value of OPN and AFP.

In our meta-analysis, we evaluated the accuracy of serum AFP and OPN+AFP for diagnosing HCC. Although only 5 of the 12 studies reported the accuracy of OPN+AFP for diagnosing HCC, the results of these studies were inconsistent. ${ }^{15,17,33,35,36}$ Our meta-analysis showed that the pooled sensitivity of OPN was higher than that of AFP, 

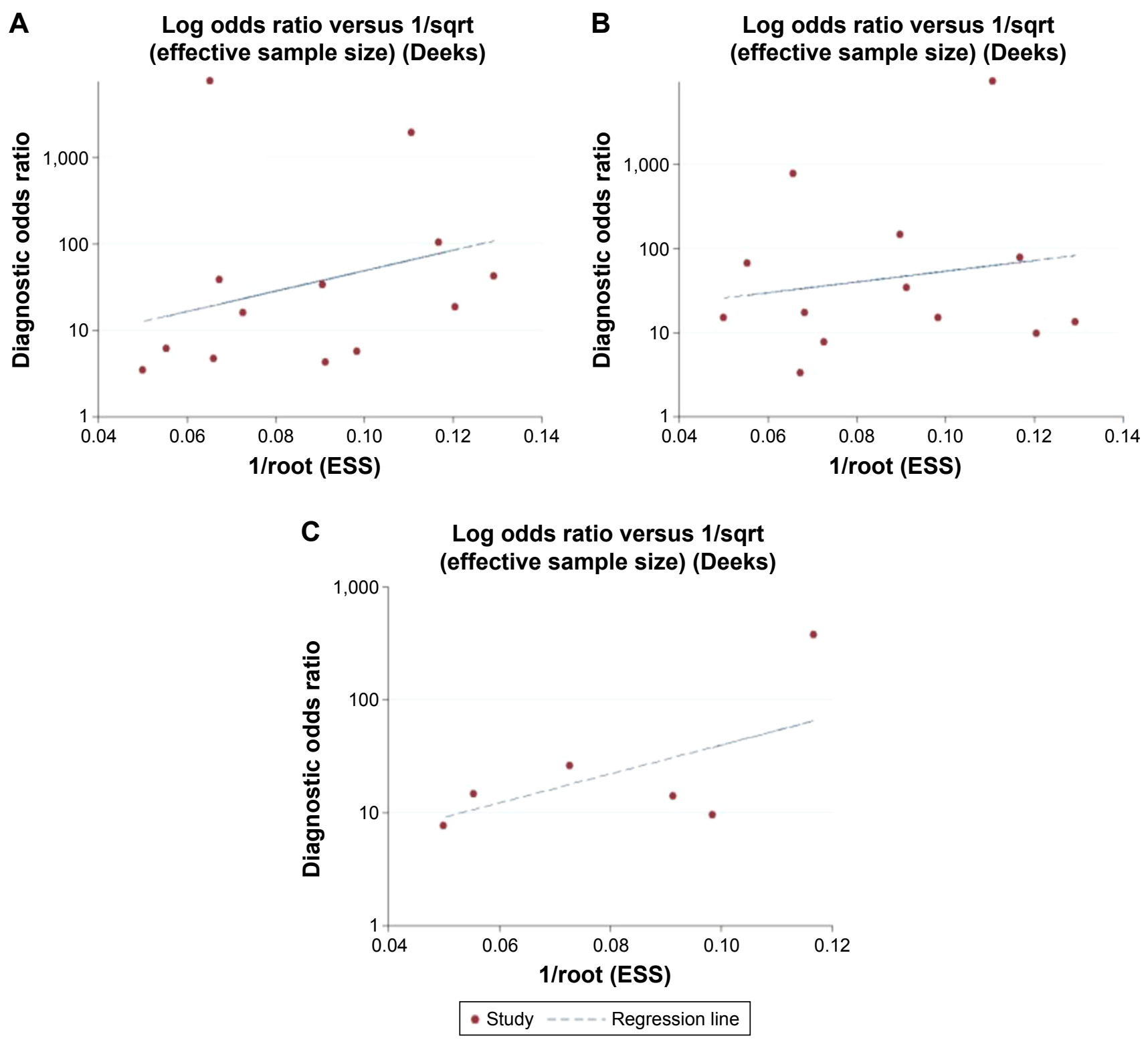

Figure 9 Begger's funnel plot of publication bias on the pooled diagnostic odds ratio of (A) OPN, (B) AFP, and (C) OPN+AFP for diagnosing hepatocellular carcinoma. Note: No publication bias was detected for this meta-analysis (Deeks' test $P=0.41$ I for AFP, $P=0.565$ for OPN, $P=0.149$ for OPN+AFP).

Abbreviations: AFP, alpha-fetoprotein; ESS, effective sample size; OPN, osteopontin; sqrt, square root.

and the sensitivity value of OPN+AFP was superior to that of OPN or AFP alone, but the specificity shows no significant differences. The mean DOR value of OPN, AFP, and OPN+AFP was 30.047, 41.518, and 16.718, respectively, suggesting there is no superiority when comparing serum levels of OPN+AFP with OPN or AFP alone. The AUC is a useful and extensive index for the sROC curve and also shows very stable performance in the heterogeneity tests. In our meta-analysis, the AUC value of OPN, AFP, and OPN+AFP was $0.91,0.88$, and 0.85 , respectively, which indicates that serum levels of OPN have higher accuracy in HCC diagnosis than those of AFP or OPN+AFP. For diagnosis of early HCC, pooled sensitivity of serum OPN, AFP, and OPN+AFP was $0.493,0.517$, and 0.732 , respectively. This result shows that the sensitivity of OPN combined with AFP is elevated compared with OPN or AFP alone. However, for HCC patients, timely and accurate diagnosis was of great value to improve the prognosis and overall survival. In order to avoid missed diagnosis for $\mathrm{HCC}$, sensitivity was usually highlighted rather than specificity in clinical application. Thus, the diagnostic value of OPN combined with AFP for HCC was better than each single marker. Our meta-analysis indicated that the diagnostic accuracy of OPN+AFP was unsatisfactory, which may due to the low amount of data. Thus, further observation is needed in more studies. 
We could not assess the pooled sensitivity and specificity by STATA as only four studies reported the diagnostic accuracy of serum OPN in the early stage of HCC and the conclusions were conflicting. ${ }^{15,34-36}$ So, we used Meta-Disc (version 1.4). The pooled sensitivity of serum OPN for diagnosis of early HCC was 0.493 . For AFP, the pooled sensitivity was 0.517 , while the pooled sensitivity for AFP combined with OPN was 0.732 . Combination of AFP and OPN can elevate the sensitivity of diagnosis for early HCC. The diagnostic ability of OPN for early HCC also needs further research because of limited studies.

In meta-analysis, one of the major goals is to explore the causes of heterogeneity rather than calculate a single summary measure..$^{40}$ Because a significant heterogeneity was also observed in the included studies, the meta-expression was used to explain it by exploring the study characteristics. However, we found that the differences in cutoff value, race, and assay method were not responsible for the heterogeneity, which might due to the low number of included studies. In addition, many studies lacked the key information about experiment design and complete reporting, and only few studies used direct comparisons of the diagnostic accuracy. This may also be an obstacle for clarifying the source of heterogeneity. Therefore, further well-designed studies with large sample sizes are needed to assess the diagnostic accuracy of OPN.

In this meta-analysis, we found some methodological problems that should be paid careful attention in future research. Some design deficiencies existed in the included studies. First, some included studies had no explicit inclusion and exclusion criteria for patient selection. Second, all studies are retrospective research and none of them described the process of patient selection. Third, one of the studies included healthy subjects as a control group, ${ }^{34}$ and only two studies clearly reported that HCC patients were diagnosed by histopathology. ${ }^{31,33}$ In addition, none of the studies clearly stated that the cutoff value of OPN was predesigned. Overall, the deficiencies of these designs may greatly influence the OPN diagnosis accuracy.

\section{Conclusion}

In summary, our results showed that OPN has moderate diagnostic performance and is able to serve as a comparable diagnosis marker to AFP in HCC. Also, for diagnosis of early HCC, the sensitivity of OPN combined with AFP is elevated compared with that of OPN or AFP alone. However, further large-scale studies are warranted to validate our findings.

\section{Disclosure}

The authors report no conflicts of interest in this work.

\section{References}

1. Malaguarnera G, Giordano M, Paladina I, Berretta M, Cappellani A, Malaguarnera M. Serum markers of hepatocellular carcinoma. Dig Dis Sci. 2010;55(10):2744-2755.

2. Bertino G, Ardiri A, Malaguarnera M, Malaguarnera G, Bertino N, Calvagno GS. Hepatocellualar carcinoma serum markers. Semin Oncol. 2012;39(4):410-433.

3. Kawano Y, Sasaki A, Kai S, et al. Short- and long-term outcomes after hepatic resection for hepatocellular carcinoma with concomitant esophageal varices in patients with cirrhosis. Ann Surg Oncol. 2008;15(6): 1670-1676.

4. Stefaniuk P, Cianciara J, Wiercinska-Drapalo A. Present and future possibilities for early diagnosis of hepatocellular carcinoma. World $J$ Gastroenterol. 2010;16(4):418-424.

5. Aghoram R, Cai P, Dickinson JA. Alpha-foetoprotein and/or liver ultrasonography for screening of hepatocellular carcinoma in patients with chronic hepatitis B. Cochrane Database Syst Rev. 2012;(9): CD002799.

6. Abd El Gawad IA, Mossallam GI, Radwan NH, Elzawahry HM, Elhifnawy NM. Comparing prothrombin induced by vitamin K absence-II (PIVKA-II) with the oncofetal proteins glypican-3, Alpha feto protein and carcinoembryonic antigen in diagnosing hepatocellular carcinoma among Egyptian patients. J Egypt Natl Canc Inst. 2014;26(2):79-85.

7. An C, Park MS, Jeon HM, et al. Prediction of the histopathological grade of hepatocellular carcinoma using qualitative diffusion-weighted, dynamic, and hepatobiliary phase MRI. Eur Radiol. 2012;22(8): 1701-1708.

8. Morris KL, Tugwood JD, Khoja L, et al. Circulating biomarkers in hepatocellular carcinoma. Cancer Chemother Pharmacol. 2014;74(2): 323-332.

9. Sherman M, Klein A. AASLD single-topic research conference on hepatocellular carcinoma: Conference proceedings. Hepatology. 2004;40(6):1465-1473.

10. Bandopadhyay M, Bulbule A, Butti R, et al. Osteopontin as a therapeutic target for cancer. Expert Opin Ther Targets. 2014;18(8):883-895.

11. Ashkar S, Weber GF, Panoutsakopoulou V, et al. Eta-1 (osteopontin): an early component of type-1 (cell-mediated) immunity. Science. 2000; 287(5454):860-864.

12. Shin T. Osteopontin as a two-sided mediator in acute neuroinflammation in rat models. Acta Histochem. 2012;114(8):749-754.

13. Yu TT, Han ZG, Shan L, et al. Expression of osteopontin in non-small cell lung cancer and correlative relation with microvascular density. Asian Pac J Cancer Prev. 2014;15(1):29-32.

14. Bramwell VH, Tuck AB, Chapman JA, et al. Assessment of osteopontin in early breast cancer: correlative study in a randomised clinical trial. Breast Cancer Res. 2014;16(1):R8.

15. Shang S, Plymoth A, Ge S, et al. Identification of osteopontin as a novel marker for early hepatocellular carcinoma. Hepatology. 2012; 55(2):483-490

16. da Costa AN, Plymoth A, Santos-Silva D, et al. Osteopontin and latentTGF $\beta$ binding-protein 2 as potential diagnostic markers for HBVrelated hepatocellular carcinoma. Int J Cancer. 2015;136(1):172-181.

17. Lee HJ, Yeon JE, Suh SJ, et al. Clinical utility of plasma glypican-3 and osteopontin as biomarkers of hepatocellular carcinoma. Gut Liver. 2014;8(2):177-185.

18. Jang ES, Jeong SH, Kim JW, Choi YS, Leissner P, Brechot C. Diagnostic performance of alpha-fetoprotein, protein induced by vitamin $\mathrm{k}$ absence, osteopontin, Dickkopf-1 and its combinations for hepatocellular carcinoma. PLoS One. 2016;11(3):e0151069.

19. Whiting PF, Rutjes AW, Westwood ME, et al. QUADAS-2: a revised tool for the quality assessment of diagnostic accuracy studies. Ann Intern Med. 2011;155(8):529-536. 
20. Reitsma JB, Glas AS, Rutjes AW, Scholten RJ, Bossuyt PM, Zwinderman AH. Bivariate analysis of sensitivity and specificity produces informative summary measures in diagnostic reviews. J Clin Epidemiol. 2005;58(10):982-990.

21. Deeks JJ, Macaskill P, Irwig L. The performance of tests of publication bias and other sample size effects in systematic reviews of diagnostic test accuracy was assessed. J Clin Epidemiol. 2005;58(9):882-893.

22. Duarte-Salles T, Misra S, Stepien M, et al. Circulating osteopontin and prediction of hepatocellular carcinoma development in a large European population. Cancer Prev Res. 2016;9(9):758-765.

23. Simão A, Madaleno J, Silva N, et al. Plasma osteopontin is a biomarker for the severity of alcoholic liver cirrhosis, not for hepatocellular carcinoma screening. BMC Gastroenterol. 2015;15:73.

24. Matsue Y, Tsutsumi M, Hayashi N, et al. Serum osteopontin predicts degree of hepatic fibrosis and serves as a biomarker in patients with hepatitis C virus infection. PLoS One. 2015;10(3):e0118744.

25. Hua Z, Chen J, Sun B, et al. Specific expression of osteopontin and S100A6 in hepatocellular carcinoma. Surgery. 2011;149(6):783-791.

26. Ying X, Zhao Y, Wang JL, et al. Serum anti-osteopontin autoantibody as a novel diagnostic and prognostic biomarker in patients with hepatocellular carcinoma. Oncol Rep. 2014;32(4):1550-1556.

27. Salem M, Atti SA, Raziky ME, Darweesh SK, Sharkawy ME. Clinical significance of plasma osteopontin level as a biomarker of hepatocellular carcinoma. Gastroenterology Res. 2013;6(5):191-199.

28. Kim J, Ki SS, Lee SD, et al. Elevated plasma osteopontin levels in patients with hepatocellular carcinoma. Am J Gastroenterol. 2006; 101(9):2051-2059.

29. El-Din Bessa SS, Elwan NM, Suliman GA, El-Shourbagy SH. Clinical significance of plasma osteopontin level in Egyptian patients with hepatitis C virus-related hepatocellular carcinoma. Arch Med Res. 2010; 41(7):541-547.

30. Abu El Makarem MA, Abdel-Aleem A, Ali A, et al. Diagnostic significance of plasma osteopontin in hepatitis $\mathrm{C}$ virus-related hepatocellular carcinoma. Ann Hepatol. 2011;10(3):296-305.
31. Yang L, Rong W, Xiao T, et al. Secretory/releasing proteome-based identification of plasma biomarkers in HBV-associated hepatocellular carcinoma. Sci China Life Sci. 2013;56(7):638-646.

32. Nabih MI, Aref WM, Fathy MM. Significance of plasma osteopontin in diagnosis of hepatitis $\mathrm{C}$ virus-related hepatocellular carcinoma. Arab J Gastroenterol. 2014;15(3-4):103-107.

33. Ge T, Shen Q, Wang N, et al. Diagnostic values of alpha-fetoprotein, dickkopf-1, and osteopontin for hepatocellular carcinoma. Med Oncol. 2015;32(3):59.

34. Fouad SA, Mohamed NA, Fawzy MW, Moustafa DA. Plasma osteopontin level in chronic liver disease and hepatocellular carcinoma. Hepat Mon. 2015;15(9):e30753.

35. Chimparlee N, Chuaypen N, Khlaiphuengsin A, et al. Diagnostic and prognostic roles of serum osteopontin and osteopontin promoter polymorphisms in Hepatitis B-related hepatocellular carcinoma. Asian Pac J Cancer Prev. 2015;16(16):7211-7217.

36. Vongsuvanh R, van der Poorten D, Iseli T, Strasser SI, McCaughan GW, George J. Midkine increases diagnostic yield in AFP negative and NASH-related hepatocellular carcinoma. PLoS One. 2016;11(5): e0155800.

37. Cleophas TJ, Zwinderman AH. Meta-analyses of diagnostic studies. Clin Chem Lab Med. 2009;47(11):1351-1354.

38. Cheng J, Wang W, Sun C, Li M, Wang B, Lv Y. Meta-analysis of the prognostic and diagnostic significance of serum/plasma osteopontin in hepatocellular carcinoma. J Clin Gastroenterol. 2014;48(9):806-814.

39. Wan HG, Xu H, Gu YM, Wang H, Xu W, Zu MH. Comparison osteopontin vs AFP for the diagnosis of HCC: a meta-analysis. Clin Res Hepatol Gastroenterol. 2014;38(6):706-714.

40. Petitti DB. Approaches to heterogeneity in meta-analysis. Stat Med. 2001;20(23):3625-3633.
OncoTargets and Therapy

\section{Publish your work in this journal}

OncoTargets and Therapy is an international, peer-reviewed, open access journal focusing on the pathological basis of all cancers, potential targets for therapy and treatment protocols employed to improve the management of cancer patients. The journal also focuses on the impact of management programs and new therapeutic agents and protocols on

\section{Dovepress}

patient perspectives such as quality of life, adherence and satisfaction. The manuscript management system is completely online and includes a very quick and fair peer-review system, which is all easy to use. Visit http://www.dovepress.com/testimonials.php to read real quotes from published authors. 